\title{
Determination of Picogram Levels of Diacerein in a Pharmaceutical Formulation by Flow-Injection Chemiluminescence
}

\author{
Suya Gao, Binghong Ma, Pengfei Ma, Yun Zhang, Kaidi Huang and Chen Huang \\ School of Pharmacy, Xi'an Medical University, Xi'an 710021, China
}

\begin{abstract}
A simple, sensitive and inexpensive method for determination of diacerein by flow-injection chemiluminescence was proposed, based on the quenching effect of diacerein on the luminol-protein (bovine serum albumin, BSA) reaction. It was found that the decrement of CL intensity was linearly proportional to the logarithm of diacerein concentration ranging from 5.0 to $7.0 \times 103 \mathrm{pg} \cdot \mathrm{mL}-1(\mathrm{r}=0.9968)$, with the limit of detection (LOD) of $1.0 \mathrm{pg} \cdot \mathrm{mL}-1(3 \sigma)$. The proposed procedure was successfully applied to the determination of diacerein in pharmaceutical formulation, human saliva, and serum samples without interference from its potential impurities, with the recoveries ranging from $96.4 \%$ to $104.0 \%$ and the relative standard deviations (RSDs) less than $4.0 \%(\mathrm{n}=6)$.
\end{abstract}

Keywords. diacerein, luminol, chemiluminescence, folw injection.

\section{Introduction}

Diacerein, named 1,8-diacetoxy-3-carboxyanthraquinone in chemical field, is a novel anti-inflammatory drug with pharmacological properties different from those of classical nonsteroidal anti-inflammatory drugs. Clinical studies have suggested that diacerein exerts a beneficial effect on the symptomatic treatment of osteoarthritis [1,2]. It is also known to inhibit the production of interleukin-1 and to prevent cartilage breakdown in a mouse granuloma model, and to slow the progression of cartilage lesions in a canine model of osteoarthritis [3,4]. Diacerein is known to be completely metabolized by animals and humans into rhein, an active metabolite of diacerein found in plasma and synovial fluid. Moreover, some researchers have related the use of diacerein or rhein to treat and prevent vascular diseases[5].

Several methods have been developed for determining diacerein such as high performance liquid chromatography, UV-vis spectrophotometry, flow-injection Chemiluminescence (FI-CL), etc [6-10]. Compared with other methods, FI$\mathrm{CL}$ is an attractive detection method for determination because of the amazing advantages of ultra sensitivity, very low detection limit, rapidity, and wide linear working range that can be achieved by use of relatively simple instrumentation. Luminol as one of the highest quantum yield luminescent compounds, has been widely used in bioluminescent and chemiluminescent analysis. In recent years, the luminol-protein system has been widely used to determine some organic compounds in pharmaceutical preparations based on their enhancing effect on this CL reaction [11]. Bovine serum albumin (BSA) is one of protein, which has been commonly used as a model protein to study protein-drug interactions. To the best of our knowledge, no detailed investigation of diacerein by luminol-protein CL system has so far been reported. In this paper, it was found that the CL intensity from luminol-protein system could be significantly inhibited in the presence of diacerein, and the decrement of CL intensity was proportional to the logarithm of diacerein concentration in wide range of 5.0 to $7.0 \times 103 \mathrm{pg} \cdot \mathrm{mL}-1$. Based on this observation, a simple, rapid and highly sensitive FI-CL method was established for the determination of diacerein in pharmaceutical formulations and biofluids with satisfactory results.

\section{Experiment}

Reagents. Luminol of $2.5 \times 10-2 \mathrm{~mol} \cdot \mathrm{L}-1$ was prepared by dissolving $0.44 \mathrm{~g}$ luminol (Sigma) in $100 \mathrm{~mL}$ of $0.01 \mathrm{~mol} \cdot \mathrm{L}$ $1 \mathrm{NaOH}$ solution in a brown calibrated flask. The stock solution of BSA (Sigma, MW 68kDa) with the concentration of 5.0 $\times 10-9 \mathrm{~mol} \cdot \mathrm{L}-1$ was prepared with purified water. All the reagents were of analytical grade and all solutions were prepared with doubly distilled water. The stock solution of diacerein (Xi'an Sino-Herb Bio-technology Co.Ltd., China) of $2.0 \times 10-4 \mathrm{mg} \cdot \mathrm{mL}-1$ was prepared with HPLC-grade methanol up to $50 \mathrm{~mL}$ in the ultrasonic generator for $60 \mathrm{~min}$ and diluted with water to prepare working solutions.

Apparatus and Procedures. The FI-CL apparatus consists of the sampling system, CL detector (photomultiplier tube), and the PC with a client system. Two peristaltic pumps (IFIS-C, Xi'an Ruike Electronic Science-Tech. Co. Ltd., China), equipped with an automatic sampler, were used to deliver all solutions at a flow rate of $2.0 \mathrm{~mL} \cdot \mathrm{min}-1$. PTFE tubing $(0.8 \mathrm{~mm}$ i.d.) was used to connect all components in the flow system. Injection was made using a six-way 
injection valve equipped with a $100 \mu \mathrm{L}$ sample loop. The flow cell was a $10.0 \mathrm{~cm}$ length of glass tubing (2.0mm i.d.) and was positioned in front of the detection window of the CR-150 photomutiplier tube (PMT, operated at -700V, Hamamatsu, Tokyo, Japan). The CL emission produced in the flow cell was detected by using a computerized BPCL ultra-weak luminescence analyzer (Institute of Biophysics, Acade-mia Sinica, Beijing, China). Data acquisition and treatment were performed with BPCL software. Four flow lines were inserted into the solutions of luminol, carrier (purified water), BSA and sample, respectively, and the solutions were propelled by peristaltic pumps. Luminol (100 $\mu \mathrm{L})$ was quantitatively injected into the mixed solution of BSA and sample by a six-way valve, and then the mixture was delivered into the flow cell producing CL emission. The signal was displayed and integrated for a $0.1 \mathrm{~s}$ interval. The concentration of the diacerein was quantified by the decrement of CL intensity $(\Delta \mathrm{I}=\mathrm{I} 0-\mathrm{Is})$, where $\mathrm{I} 0$ and Is were CL signals in the absence and in the presence of diacerein, respectively.

Sample Preparation. Diacerein capsules (labelled amount 50mg-cap-1) purchased from local market, were disintegrated, weighed and finely powdered. Then the powder of average one capsule was accurately weighed and transferred into a $100 \mathrm{~mL}$ volumetric flask, and then diluted to volume with HPLC-grade methanol. Then, it was placed in an ultrasonic generator for $1 \mathrm{~h}$ and the resulting mixture was filtered. The filtrate was further diluted with water so that the final diacerein concentration was within the working range, and then analyzed according to the procedure described above. The saliva was collected from healthy volunteers, and the serum was supplied by the Shaanxi Province People's Hospital. To prepare the spiked samples, known quantities of standard solution of diacerein were spiked into $1.0 \mathrm{~mL}$ of saliva or serum. After homogenization, the spiked samples with appropriate dilution were taken for the quantification.

\section{Results and Discussion}

Optimization of Luminol-BSA CL System. Under different concentrations of luminol solution from $1.0 \times 10-8 \mathrm{~mol} \cdot \mathrm{L}-1$ to $1.0 \times 10-4 \mathrm{~mol} \cdot \mathrm{L}-1$ and BSA solution from $1.0 \times 10-11 \mathrm{~mol} \cdot \mathrm{L}-1$ to $5.0 \times 10-7 \mathrm{~mol} \cdot \mathrm{L}-1$, the CL intensity was determined. It was found that the CL intensity arrived at the maximum with $1.0 \times 10-5 \mathrm{~mol} \cdot \mathrm{L}-1$ lumimol and $5.0 \times 10-9 \mathrm{~mol} \cdot \mathrm{L}-1 \mathrm{BSA}$ (shown in Fig.1a). $\mathrm{NaOH}$ was introduced into the luminol solution to improve the sensitivity of the system, and $0.01 \mathrm{~mol} \cdot \mathrm{L}-1 \mathrm{NaOH}$ was the optimal in the experiment (shown in Fig. $1 \mathrm{~b}$ ). In addition, the influence of flow rate and the length of mixing tube on the CL intensity were also investigated in the ranges of $0.5 \sim 5 \mathrm{~mL} \cdot \mathrm{min}-1$ and $5.0 \sim 20.0 \mathrm{~cm}$. As a compromise between good precision and lower reagent consumption, the flow rate of $2.0 \mathrm{~mL} \cdot \mathrm{min}-1$ on each flow line and the length of $10.0 \mathrm{~cm}$ mixing tube afforded the best results with good sensitivity and reproducibility.
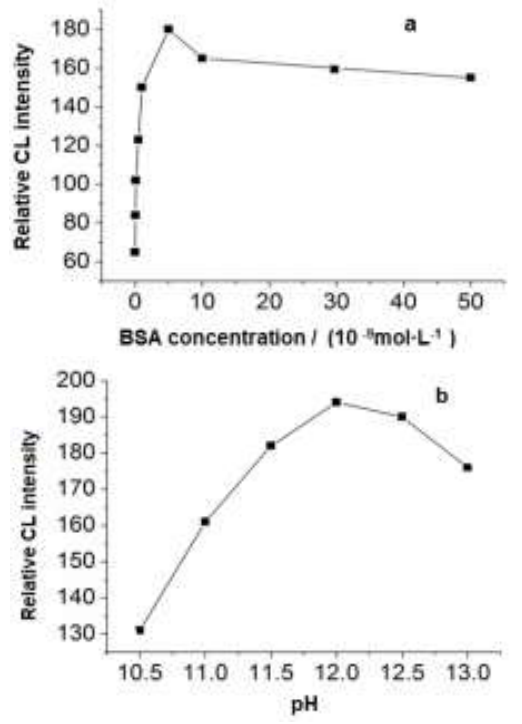

Fig.1 Effect of BSA concentration (a) and pH (b) on the CL intensity

Standard Curve and Detection Limit. Under the selected conditions described above, the calibration graph of CL decrement $(\Delta \mathrm{I}$, relative unit) versus the logarithm of diacerein concentration was linear in the range of $5.0 \sim 7.0 \times 103$ $\mathrm{pg} \cdot \mathrm{mL}-1$ with a detection limit of $1.0 \mathrm{pg} \cdot \mathrm{mL}-1(3 \sigma)$. The regression equation was $\Delta \mathrm{I}=38.0 \operatorname{lgC}-27.6$ with a correlation coefficient of $0.9968(\mathrm{n}=6)$. The relative standard deviation was $2.2 \%$ for $50 \mathrm{pg} \cdot \mathrm{mL}-1 \mathrm{diacerein}$ in 8 replicate measurements. At flow rate of $2.0 \mathrm{~mL} \cdot \mathrm{min}-1$, both sampling and washing could be accomplished in $30 \mathrm{~s}$. So the sample measurement frequency was calculated about 120 samples $\cdot h-1$. Repeated injection of standards and samples leads to easily reproducible data. It indicated that FI-CL was a non-time consuming strategy for the quantification of diacerein. 
Interference. In order to assess the possible analytical application of the proposed method to dosage forms, the effect of foreign substances was studied by analyzing a standard solution of $50 \mathrm{pg} \cdot \mathrm{mL}-1$ diacerein to which increasing amounts of interfering species and potential interfering substances were added. Assuming interference at a level of $\pm 5 \%$, tolerable ratios of foreign species with respect to $5.0 \times 102 \mathrm{pg} \cdot \mathrm{mL}-1$ diacerein were over $1.0 \times 104$ times for the excipients commonly existed in the capsules, such as glucose, sucrose, starch, methanol, ethanol and fructose; $2.0 \times 103$ for $\mathrm{Na}+, \mathrm{K}+, \mathrm{Ba} 2+, \mathrm{Mg} 2+, \mathrm{Ca} 2+, \mathrm{NH} 4+, \mathrm{F}-, \mathrm{Cl}-, \mathrm{Br}-, \mathrm{I}-, \mathrm{SO} 42-, \mathrm{NO} 3-, \mathrm{CO} 32-; 1.0 \times 103$ for Zn2+, Ni2+, $\mathrm{Mn} 2+, \mathrm{Cd} 2+$, $\mathrm{Ag}+, \mathrm{Cu} 2+, \mathrm{Hg} 2+; 1.0 \times 102$ for the tryptophan, tyrosine, dopamine and peroxidase. In fact, the effect of above species and some other foreign substances in saliva and serum can be eliminated by calibrating the blank prior to diacerein determination. Thus the influence on the determination of diacerein at picogram levels can be neglected under the experimental conditions.

Analysis of Real Sample. Following the procedure described previously, the proposed CL method was applied to the determination of diacerein in commercially available Artrodar (diacerein capsules, labeled $50 \mathrm{mg}$ per capsule). The results were listed in Table 1, and each result was the average of six determinations and agreed well with the labeled values. It can be seen that the recoveries were from $97.8 \%$ to $101.2 \%$ and RSDs were less than $3.0 \%(n=6)$.

Determination of diacerein in human saliva and serum samples. To validate the expanded applicability of the method for the determination of diacerein in the complex biological matrix, the spiked human saliva and serum samples prepared in the experimental section were quantified, and the results were listed in Table 2 and, Table 3, respectively. Each result was the average of five determinations. It was clear that the recoveries ranged from $96.4 \%$ to $104.0 \%$ and RSDs were less than $4.0 \%(n=6)$. A good agreement between the added and found concentration values was achieved. The results proved that the proposed method could be applied to the determination of diacerein in biofluids.

Table 1. Results for the determination of diacerein in Artrodar capsules

\begin{tabular}{ccccc}
\hline Sample & $\begin{array}{c}\text { Proposed method } \\
\mathbf{a}\left(\mathbf{m g} \mathbf{c a p}^{-\mathbf{1}}\right)\end{array}$ & $\begin{array}{c}\text { Added } \\
\left(\mathbf{p g} \mathbf{m L}^{-\mathbf{1}}\right)\end{array}$ & $\begin{array}{c}\text { Recovered } \\
\left(\mathbf{p g} \mathbf{m L}^{\mathbf{- 1}}\right)\end{array}$ & $\begin{array}{c}\text { average } \\
\text { recovery (\%) }\end{array}$ \\
\hline 1 & $49.39 \pm 1.3$ & 10.00 & $10.12 \pm 2.8$ & 101.2 \\
2 & $50.02 \pm 1.8$ & 20.00 & $19.57 \pm 1.9$ & 97.8 \\
3 & $49.65 \pm 2.6$ & 30.00 & $30.08 \pm 2.3$ & 100.3 \\
4 & $49.50 \pm 2.9$ & 20.00 & $19.92 \pm 2.6$ & 99.6 \\
5 & $49.18 \pm 2.1$ & 40.00 & $40.19 \pm 2.4$ & 100.5 \\
6 & $49.89 \pm 1.9$ & 60.00 & $58.93 \pm 2.1$ & 98.2 \\
\hline
\end{tabular}

Table 2. Results for the determination of diacerein in spiked saliva

\begin{tabular}{ccccc}
\hline Sample & Added $\left(\mathbf{p g} \mathbf{m L}^{-\mathbf{1}}\right)$ & Found $\left(\mathbf{p g} \mathbf{m L}^{-\mathbf{1}}\right)$ & $\mathbf{R S D}(\%)$ & Recovery(\%) \\
\hline 1 & $0 / 20.0$ & $50.1 / 70.2$ & $3.1 / 2.5$ & 100.5 \\
2 & $0 / 50.0$ & $50.5 / 101.8$ & $3.6 / 3.2$ & 102.6 \\
3 & $0 / 100.0$ & $49.6 / 150.4$ & $2.3 / 1.9$ & 100.8 \\
4 & $0 / 200.0$ & $49.4 / 242.2$ & $2.8 / 2.6$ & 96.4 \\
5 & $0 / 300.0$ & $50.2 / 349.6$ & $3.4 / 2.8$ & 99.8 \\
6 & $0 / 500.0$ & $49.8 / 558.5$ & $2.5 / 1.7$ & 101.7 \\
\hline
\end{tabular}

Table 3. Results for the determination of diacerein in spiked serum

\begin{tabular}{ccccc}
\hline Sample & Added(pg m $\left.\mathbf{L}^{-1}\right)$ & Found $\left(\mathbf{p g} \mathbf{m L}^{-\mathbf{1}}\right)$ & RSD(\%) & Recovery(\%) \\
\hline 1 & $0 / 10.00$ & $20.8 / 31.5$ & $2.6 / 3.5$ & 107.0 \\
2 & $0 / 20.00$ & $20.1 / 40.9$ & $2.1 / 3.6$ & 104.0 \\
3 & $0 / 50.00$ & $20.6 / 71.2$ & $3.1 / 2.8$ & 101.2 \\
4 & $0 / 100.00$ & $19.8 / 118.5$ & $2.9 / 2.2$ & 98.7 \\
5 & $0 / 200.00$ & $19.2 / 220.8$ & $3.2 / 3.7$ & 100.8 \\
6 & $0 / 300.00$ & $19.5 / 317.4$ & $2.4 / 3.0$ & 99.3 \\
\hline
\end{tabular}




\section{Conclusions}

A simple, sensitive and rapid flow injection CL method of luminol-BSA reaction in alkaline medium for the determination of diacerein was developed for the first time. Even with a simple set-up, diacerein can be sensitively detected with a $1.0 \mathrm{pg} \cdot \mathrm{mL}-1$ detection limit $(3 \sigma)$. Moreover, the proposed procedure was successfully applied to the determination of diacerein in pharmaceutical formulation, human saliva, and serum samples without interference from its potential impurities. Satisfactory performance of the assay of diacerein in pharmaceutical preparations and biofluids has proved the ability of this method for analyzing complex biological matrix with separation-free, indicating its potential value in pharmaceutical and clinic researches.

\section{Acknowledgments}

This work was supported by the Undergraduates' Innovative Training Program (No. 1647, 2015DXS1-22), the Ph.D. Startup Foundation of Xi' an Medical University of China (No.2012DOC12), the Scientific Research Program Funded by Shaanxi Provincial Education Department (No. 14JK1620) and the discipline construction of Xi'an Medical University.

\section{References}

1. Tamura, T. Shirai, T. Kosaka, N. Ohmori, K. Takafumi, N. Eur. J. Pharmacol, 448 (2002)

2. Pelletier, J.P. Yaron, M. Haraoui, B. Cohen, P. Nahir, M.A. Choquette, D. Wigler, I. Rosner, I.A.; Beauleu, A.D. Arthritis Rheum, 43 (2000)

3. Cui-e He, Y.M. Wang, Journal of clinical research. 28 (2011)

4. S Singh, A Jain, S K Mishra, et al. Osteoarthr Cartilage, 20 (2012)

5. J.-M. Lin, Yamada, M. Trends Anal, Chem, 22 (2003)

6. Giannellini, V. Salvatore, F. Bartolucci, G. Coran, S.A. Bambagiotti-Alberti, M. J. Pharm, Biomed. Anal. 39 (2005)

7. S Dhaneshwar, V Patel, D Patil. Bioorg, Med. Chem. Lett, 23 (2013)

8. H.C. Yao, X.F. Yang, H. Li, J. Chin, Chem.Soc, 54 (2007)

9. S Bais, I Singhvi, A Chandewar, M Shirsat, Asian J. Pharm. Ana, 3 (2013)

10. K. R. Sreejith, Dr. K. Premalatha, International J. Research in Pharm, Biomed. Sci. 2 (2011)

11. Y. Zhang, S.Y. Gao, Advanced Material Research, 884-885 (2014) 